\title{
A CROSS-CULTURAL STUDY ON WHITE COLOUR IDIOMS IN TURKISH AND ENGLISH: CONCEPTUAL METAPHOR THEORY IN FOCUS
}

\author{
Gökçen Hastürkoğlu**
}

\begin{abstract}
This study aims at investigating how similar and different the embodied cognition of Turkish and English speakers is by providing a systematic description of Turkish and English white colour idiomatic expressions and by analyzing them within the framework of Conceptual Metaphor Theory through which the cognitive motivations behind the idiomatic expressions can be demonstrated. In order to do so, a large-scale corpus study based on specialized dictionaries on idioms in Turkish and English was carried out and a table was presented for each language illustrating the idiomatic expression, its meaning, its translation for the Turkish part, and the underlying conceptual metaphor or metonymy. After this cognitive analysis, it was revealed that despite some similarities in the cognitive mappings of the idioms in Turkish and English, the connotations of white colour idioms in two genetically unrelated languages vary because of cultural, historical, religious, or customary matters.
\end{abstract}

Key words: white colour, idiomatic expressions, conceptual metaphor, conceptual metonymy, cultural cognition

\section{Introduction}

With the advent of cognitive perspectives of metaphors in 1980s, the long standing idea supporting the fact that metaphors are one of the components of stylistic language was abandoned. Since then, more and more researchers have focused on the metaphors as a tool in human communication. In Metaphors We Live By, Lakoff and Johnson revealed the metaphorical structure of human mind and stressed that meaning making is a process of structuring abstract concepts in terms of more concrete concepts (1980: 109). They emphasized that "Our concepts structure what we perceive, how we get around in the world, and how we relate to other people. Our conceptual system; thus, plays a central role in defining our everyday realities" (ibid.: 3 ). They provided the conceptual

\footnotetext{
* This study is based on a part of the PhD Thesis of the researcher titled "A Corpus-Based Cognitive Study on the Comparison of Collocational Realizations of Basic Colour Terms in Turkish and English" written under the supervision of Prof. Dr. Işı1 Özyıldırım at Hacettepe University.

** Lecturer at Atılım University, Ankara, Turkey, e-mail: gokcen.hasturkoglu@atilim.edu.tr
} 
metaphor ARGUMENT IS WAR as an example. In statements like "He attacked every weak point in my argument", "Your claims are indefensible", and "I demolished his argument", the source domain WAR has entities such as position, combatant, allies, etc., while the target domain has entities such as opinion, debate participant, agreement, etc., and people are directed to talk and think about the target domain ARGUMENT in terms of the source domain WAR. (ibid.: 4). On the other hand, they mentioned the possibility that there may be different cultures in which arguments are not thought in terms of war, but viewed as a dance (ibid.: 5); therefore, in such cultures in which arguments are conceptualized as a dance, instead of ARGUMENT IS WAR, the underlying conceptual metaphor is ARGUMENT IS DANCE (ibid.: 5). As the experiences and perceptions of individuals in different cultures vary, their conceptualizations or their associating abstract things with the concrete ones change accordingly.

In Metaphor in Cognitive Linguistics the importance of culture in the studies on metaphor was emphasized by asserting that in such studies there is the requirement of "an explicit acknowledgment of culture and its important, perhaps defining, role in shaping embodiment and, consequently metaphorical thought" (Gibbs, Steen 1997: 153). Relatedly, Lakoff and Johnson discussed the relationship between culture and metaphor as follows: "The most fundamental values in a culture will be coherent with the metaphorical structure of the most fundamental concepts in culture" (1980: 22).

As a thematic and representative group of metaphorical language, colours can be regarded as the most prominent aspects of culture and colour terms have been studied within the fields of linguistics, cognitive, cultural, and translation studies. In this study, idioms which are constructed around the colour white, one of the basic colour terms as Berlin and Kay (1969) put it, were elaborated in order to shed light on the similarities and dissimilarities of collocational realizations of the colour white in Turkish and English idioms by providing the socio-cultural motivation behind these conceptualizations.

\section{Previous Research in the Field}

A number of comparative studies investigating the idioms with basic colour terms from a cognitive point of view can be seen in the literature such as "Connotative Meaning in English and Italian Colour-Word Metaphors" by Philip (2006) in which she revealed the frequencies of the basic colour terms in English and Italian and emphasized that connotations of colours are not universal and that the associated meanings of colours change greatly among different cultures. Another important study is "Basic Colour Terms in English: An Examination of Their Use and Meaning in English Expressions" (Chielens 2007) which aimed at demonstrating why certain colour terms are used in expressions and what 
meanings can be expressed by them. Although Chielens included the examples of metaphorical expressions of colour terms in Dutch, this study cannot be called bilingual work; as there are only several examples provided in some chapters to compare with the English expressions. "A Comparative Study of Color Metaphors in English and Chinese" by He (2011) is a study which revealed the similarities and differences between the conceptual metaphors of colour expressions including black, white, red, yellow, blue in Chinese and English. The metaphorical expressions of colour terms were selected randomly; thus, not all metaphorical expressions were included in the study. "Basic Colors and Their Metaphorical Expressions in English and Persian: Lakoff's Conceptual Metaphor Theory in Focus" (Rasekh, Ghafel 2011) is another comparative study in which the purpose was to investigate the connotations of basic colours in English and Persian and reveal the differences between these two languages. Rasekh and Ghafel compiled metaphoric expressions of colours from different dictionaries and conducted the analysis of some of the expressions, which they regarded as influential, to demonstrate cultural variations and similarities between the English and the Persian society.

While reviewing the literature, it was observed that the number of crosscultural studies conducted on the analysis of basic colour terms is low in Turkey. "Fransızca ve Türkçe Renk İsimlerini İçeren Deyimlerin Karşılaştırmalı İncelenmesi" (A Comparative Analysis of the French and Turkish Idioms with Colour Names) by Topçu (2001) is a study providing a very limited corpus and trying to give a general perspective of the connotation of all the basic colours in Turkish and French. However, while presenting the similarities and variations in Turkish and French idioms with colour names, the researcher did not adopt Conceptual Metaphor Theory and did not deal with the issue on a cognitive basis. It should also be emphasized that there are monolingual studies conducted on colour terms in Turkish, but they were not described in this study.

\section{Method}

For ensuring more accurate results, the corpus was collected from a large number of specialized dictionaries of idioms. For the English corpus, Cambridge International Dictionary of Idioms, Thesaurus of Traditional English Metaphors, Oxford Dictionary of Idioms, Collins CoBuild Dictionary of Idioms, Metaphorically Speaking: A Dictionary of 3800 Picturesque Idiomatic Expressions, McGraw-Hill's Dictionary of American Idioms and Phrasal Verbs, Dictionary of Idioms and Their Origins were used. For the Turkish corpus, the Online Dictionary of Proverbs and Idioms of Turkish Language Association (TDK) and a number of printed dictionaries on idioms which were compiled by Yörük and Yörük (1997), Aksoy (1998) and the work of Eminoğlu, Türkçede Renkler Sözlüğü (Dictionary of Colours in Turkish) (2014) were scanned in this study. 
As one of the limitations of this study, the idioms which include another colour term were excluded from the database such as ak koyun kara koyun, to be a black and white issue, etc. For the remaining idioms, their usage in daily communication was found out, as it is crucial to know the contextual information of the expression in a foreign culture in order to determine the conceptual metaphors or metonymies of the expressions in question.

The analysis of the white colour idioms was presented through tables for Turkish and English separately, demonstrating the idiom, its metaphorical meaning (MM), the translation of the metaphorical meaning of the idiom for Turkish, and the conceptual metaphor/metonymy underlying the idiom which was determined within the framework of CMT. The interpretation of the tables was conducted through the instances from the recurring conceptual metaphors/ metonymies.

\section{Results and Discussion}

Table 1 and Table 2 illustrate the conceptual metaphor/metonymy in Turkish and English white colour idioms:

\begin{tabular}{|l|l|l|}
\hline \multicolumn{1}{|c|}{ Idiom } & \multicolumn{1}{|c|}{ Metaphorical Meaning (MM) } & \multicolumn{1}{c|}{$\begin{array}{c}\text { Conceptual Metaphor/ } \\
\text { Metonymy }\end{array}$} \\
\hline ak akçe & MM: nakit para (cash) & CASH IS WHITE \\
\hline ak gözlü & $\begin{array}{l}\text { MM: gözlerinin rengi açık olan } \\
\text { ve nazarının hemen değdiğine } \\
\text { inanılan kimse (blue-eyed person } \\
\text { who is likely to bring a curse) }\end{array}$ & $\begin{array}{l}\text { WHITE EYE STANDS } \\
\text { FOR EVIL }\end{array}$ \\
\hline $\begin{array}{l}\text { MM: mesut ve mutlu gün (a happy } \\
\text { day) }\end{array}$ & $\begin{array}{l}\text { MM: bembeyaz, temiz, parlak, } \\
\text { saçı sakalı ağarmış (very clean, old } \\
\text { person) }\end{array}$ & $\begin{array}{l}\text { CLEANLINESS IS } \\
\text { WHITE; OLDNESS IS } \\
\text { WHITE }\end{array}$ \\
\hline $\begin{array}{l}\text { ak pak sakaldan yok } \\
\text { sakala gelmek }\end{array}$ & $\begin{array}{l}\text { MM: çok yaşlanıp iyice kuvvetten } \\
\text { düşmek (to become very old and } \\
\text { lose strength) }\end{array}$ & $\begin{array}{l}\text { WHITE BEARD } \\
\text { STANDS FOR OLDNESS }\end{array}$ \\
\hline ak sakallı & $\begin{array}{l}\text { MM: yaşlı (old) } \\
\text { LM: white-bearded }\end{array}$ & $\begin{array}{l}\text { WHITE BEARD } \\
\text { STANDS FOR OLDNESS } \\
\text { AND WISDOM }\end{array}$ \\
\hline ak süt & $\begin{array}{l}\text { MM: namuslu kadının helal sütü } \\
\text { (honest) }\end{array}$ & HONESTY IS WHITE \\
\hline ak süt emmişs & $\begin{array}{l}\text { MM: asil, soylu, faziletli, doğru, } \\
\text { ahlâklı (as straight as a die) }\end{array}$ & HONESTY IS WHITE \\
\hline
\end{tabular}




\begin{tabular}{|c|c|c|}
\hline Idiom & Metaphorical Meaning (MM) & $\begin{array}{c}\text { Conceptual Metaphor/ } \\
\text { Metonymy }\end{array}$ \\
\hline $\begin{array}{l}\text { ak topuk beyaz } \\
\text { gerdan }\end{array}$ & $\begin{array}{l}\text { MM: çok güzel kadın (very } \\
\text { beautiful woman) }\end{array}$ & $\begin{array}{l}\text { WHITE ANKLE AND } \\
\text { WHITE NECK STAND } \\
\text { FOR BEAUTY }\end{array}$ \\
\hline ak yazl & MM: baht, şans (fortune, luck) & GOOD LUCK IS WHITE \\
\hline ak yüzlü & $\begin{array}{l}\text { MM: temiz, namuslu, doğru } \\
\text { (honest, virtuous) }\end{array}$ & $\begin{array}{l}\text { WHITE FACE STANDS } \\
\text { FOR HONESTY }\end{array}$ \\
\hline alnı açık yüzü ak & $\begin{array}{l}\text { MM: çekinecek hiçbir durumu } \\
\text { veya ayıbı olmayan (conducting } \\
\text { any dishonest behaviour) }\end{array}$ & $\begin{array}{l}\text { WHITE FACE STANDS } \\
\text { FOR HONESTY }\end{array}$ \\
\hline alninin akıyla & $\begin{array}{l}\text { MM: ayıplanacak bir duruma } \\
\text { düşmeden, şerefiyle başarı } \\
\text { göstermiş olarak (with pride, } \\
\text { honourably) }\end{array}$ & $\begin{array}{l}\text { WHITE FOREHEAD } \\
\text { STANDS FOR PRIDE }\end{array}$ \\
\hline $\begin{array}{l}\text { ananın ak sütü } \\
\text { gibi }\end{array}$ & $\begin{array}{l}\text { MM: anamın sütü bana nasıl helal } \\
\text { ise bu da sana öyle helal olsun } \\
\text { anlamında kullanılan bir söz } \\
\text { (honestly deserving something } \\
\text { without any suspicion) }\end{array}$ & HONESTY IS WHITE \\
\hline beyaz bayrak & $\begin{array}{l}\text { MM:atletizm yarışlarında } \\
\text { hakemlerce gösterilen, sporcunun } \\
\text { kurallara uygun bir biçimde } \\
\text { atladığını veya koştuğunu belirten } \\
\text { kısa saplı bayrak (white flag) }\end{array}$ & $\begin{array}{l}\text { WHITE FLAG STANDS } \\
\text { FOR PEACE }\end{array}$ \\
\hline beyaz çekmek & MM: eroin çekmek (use heroin) & $\begin{array}{l}\text { WHITE STANDS FOR } \\
\text { HEROIN }\end{array}$ \\
\hline beyaz kömür & $\begin{array}{l}\text { MM: akarsulardan elde edilen } \\
\text { elektrik gücü (electrical power } \\
\text { produced from stream) }\end{array}$ & $\begin{array}{l}\text { WHITE COAL STANDS } \\
\text { FOR ELECTRICAL } \\
\text { ENERGY }\end{array}$ \\
\hline beyaz oy & $\begin{array}{l}\text { MM: bir oylamada kabul anlamı } \\
\text { taşıyan oy (positive vote) }\end{array}$ & $\begin{array}{l}\text { WHITE VOTE STANDS } \\
\text { FOR AGREEMENT }\end{array}$ \\
\hline beyaz ölüm & $\begin{array}{l}\text { MM: aşırı ölçüde alınan eroinin } \\
\text { yol açtı̆g ölüm (death because of } \\
\text { excessive amount of heroin) }\end{array}$ & $\begin{array}{l}\text { WHITE STANDS FOR } \\
\text { HEROIN }\end{array}$ \\
\hline $\begin{array}{l}\text { beyaz sayfa } \\
\text { açmak }\end{array}$ & $\begin{array}{l}\text { MM: bir konuda geçmişi unutarak } \\
\text { geleceğe umutla bakmak (to turn } \\
\text { over a new leaf) }\end{array}$ & HOPE IS WHITE \\
\hline beyaz Türkçe & $\begin{array}{l}\text { MM: açık ve anlaşılır Türkçe (pure } \\
\text { Turkish) }\end{array}$ & PURITY IS WHITE \\
\hline
\end{tabular}




\begin{tabular}{|c|c|c|}
\hline Idiom & Metaphorical Meaning (MM) & $\begin{array}{c}\text { Conceptual Metaphor/ } \\
\text { Metonymy }\end{array}$ \\
\hline beyaz yakall & $\begin{array}{l}\text { MM: üretim sürecinde bedensel } \\
\text { gücüyle çalışmayıp düşünsel } \\
\text { etkinlikte bulunan, maaş veya } \\
\text { ücret karş1lığında çalışan memur, } \\
\text { teknik personel (white collar, a } \\
\text { worker or officer engaging in non- } \\
\text { manual work, technical personnel) }\end{array}$ & $\begin{array}{l}\text { WHITE COLLAR } \\
\text { STANDS FOR PERSON } \\
\text { PERFORMING NON- } \\
\text { MANUAL WORK }\end{array}$ \\
\hline beyaz yalan & $\begin{array}{l}\text { MM: karşısındakini üzmemek } \\
\text { veya zarar vermemek için söylenen } \\
\text { masumca yalan (white lie) }\end{array}$ & $\begin{array}{l}\text { HARMLESSNESS IS } \\
\text { WHITE }\end{array}$ \\
\hline beyaza çekmek & $\begin{array}{l}\text { MM: yazıyı temize çekmek (to } \\
\text { make a fair copy) }\end{array}$ & $\begin{array}{l}\text { WHITE STANDS FOR A } \\
\text { CLEAN PAGE }\end{array}$ \\
\hline beyaza çıkarmak & $\begin{array}{l}\text { MM: Temize çıkarmak (to be } \\
\text { purified) }\end{array}$ & $\begin{array}{l}\text { WHITE STANDS FOR } \\
\text { PURIFICATION }\end{array}$ \\
\hline $\begin{array}{l}\text { beyazlara } \\
\text { bürünmek }\end{array}$ & $\begin{array}{l}\text { MM: her yan karlarla kaplı olmak; } \\
\text { beyaz elbiseler giymek (to be } \\
\text { covered with snow, to wear white) }\end{array}$ & $\begin{array}{l}\text { WHITE STANDS FOR } \\
\text { WEARING WHITE } \\
\text { CLOTHES AND SNOW }\end{array}$ \\
\hline beyaz zehir & $\begin{array}{l}\text { MM: eroin,kokain gibi toz } \\
\text { durumunda olan uyuşturucu } \\
\text { madde (drugs such as heroin, } \\
\text { cocaine) }\end{array}$ & $\begin{array}{l}\text { WHITE STANDS FOR } \\
\text { HEROIN }\end{array}$ \\
\hline gözünü ağartmak & $\begin{array}{l}\text { MM: gözlerini belertmek, öfkeyle, } \\
\text { akı görünecek şekilde gözlerini } \\
\text { açmak, çok kızmak (to wide open } \\
\text { the eye, with anger) }\end{array}$ & $\begin{array}{l}\text { WHITE EYE STANDS } \\
\text { FOR ANGER }\end{array}$ \\
\hline gün ă̆artmak & MM: tan yeri aydınlanmak (dawn) & LIGHT IS WHITE \\
\hline kar beyaz & $\begin{array}{l}\text { MM: bembeyaz, çok beyaz (very } \\
\text { white) }\end{array}$ & $\begin{array}{l}\text { CLEANLINESS IS } \\
\text { WHITE }\end{array}$ \\
\hline ortalık ăgarmak & $\begin{array}{l}\text { MM: sabah olmaya başlamak } \\
\text { (dawn) }\end{array}$ & LIGHT IS WHITE \\
\hline saç ağartmak & $\begin{array}{l}\text { MM: saç sakal ağartmak, o işte } \\
\text { uzun zaman çalışmış, emek vermiş } \\
\text { olmak (to work on and struggle for } \\
\text { sth.) }\end{array}$ & $\begin{array}{l}\text { WHITE HAIR STANDS } \\
\text { FOR OLDNESS AND } \\
\text { BEING EXPERIENCED }\end{array}$ \\
\hline $\begin{array}{l}\text { saçı (saçları) } \\
\text { değirmende } \\
\text { ağartmamak }\end{array}$ & $\begin{array}{l}\text { MM: deneyimli olmak (to be } \\
\text { experienced) }\end{array}$ & $\begin{array}{l}\text { WHITE HAIR } \\
\text { STANDS FOR BEING } \\
\text { EXPERIENCED }\end{array}$ \\
\hline saçı başı ağarmak & MM: yaşlanmak (to become old) & $\begin{array}{l}\text { WHITE HAIR AND } \\
\text { WHITE FACE STAND } \\
\text { FOR OLDNESS } \\
\end{array}$ \\
\hline saçına ak düşmek & $\begin{array}{l}\text { MM: saçı ağarmaya başlamak, } \\
\text { yaşlanmak (to turn grey) }\end{array}$ & $\begin{array}{l}\text { WHITE HAIR STANDS } \\
\text { FOR OLDNESS }\end{array}$ \\
\hline
\end{tabular}




\begin{tabular}{|c|c|c|}
\hline Idiom & Metaphorical Meaning (MM) & $\begin{array}{c}\text { Conceptual Metaphor/ } \\
\text { Metonymy }\end{array}$ \\
\hline $\begin{array}{l}\text { Şakakları } \\
\text { ăgarmak } \\
\text { (beyazlanmak) }\end{array}$ & MM: yaşlanmak (to become old) & $\begin{array}{l}\text { WHITE TEMPORAL } \\
\text { STANDS FOR OLDNESS }\end{array}$ \\
\hline $\begin{array}{l}\text { sakall } \\
\text { dĕ̌irmende } \\
\text { ăgartmak }\end{array}$ & $\begin{array}{l}\text { MM: yıllar pek çok deneyim } \\
\text { kazandırmış olmak (to be } \\
\text { experienced) }\end{array}$ & $\begin{array}{l}\text { WHITE BEARD } \\
\text { STANDS FOR BEING } \\
\text { EXPERIENCED } \\
\end{array}$ \\
\hline $\begin{array}{l}\text { sakalına ak } \\
\text { düşmek }\end{array}$ & $\begin{array}{l}\text { MM: sakalı ağarmaya başlamak, } \\
\text { yaşlanmak (to become old) }\end{array}$ & $\begin{array}{l}\text { WHITE BEARD } \\
\text { STANDS FOR OLDNESS }\end{array}$ \\
\hline süt beyaz & $\begin{array}{l}\text { MM: bembeyaz, çok beyaz (very } \\
\text { white) }\end{array}$ & $\begin{array}{l}\text { CLEANLINESS IS } \\
\text { WHITE }\end{array}$ \\
\hline $\begin{array}{l}\text { sütten çıkmış ak } \\
\text { kaşık gibi olmak }\end{array}$ & MM: temiz, saf olmak (lily-white) & $\begin{array}{l}\text { WHITE FACE STANDS } \\
\text { FOR HONESTY }\end{array}$ \\
\hline $\begin{array}{l}\text { tan ăgarmak } \\
\text { (atmak, sökmek) }\end{array}$ & $\begin{array}{l}\text { MM: gün doğmaya başlamak, } \\
\text { şafak sökmek (dawn) }\end{array}$ & LIGHT IS WHITE \\
\hline tanyeri ă̆armak & $\begin{array}{l}\text { MM: sabah olmaya başlamak } \\
\text { (dawn) }\end{array}$ & LIGHT IS WHITE \\
\hline yüz akı & MM: övünç kaynağı (pride) & $\begin{array}{l}\text { WHITE FACE STANDS } \\
\text { FOR PRIDE }\end{array}$ \\
\hline yüzü $a k$ & $\begin{array}{l}\text { MM: suçu ve utanılacak bir } \\
\text { durumu olmayan (a person who has } \\
\text { no guilt or shame) }\end{array}$ & $\begin{array}{l}\text { WHITE FACE STANDS } \\
\text { FOR HONESTY }\end{array}$ \\
\hline $\begin{array}{l}\text { yüzü kireç gibi } \\
\text { olmak (ağarmak) }\end{array}$ & $\begin{array}{l}\text { MM: yüzünde renk kalmamak, } \\
\text { rengi solmak (to blanch) }\end{array}$ & $\begin{array}{l}\text { WHITE FACE STANDS } \\
\text { FOR PALENESS AND } \\
\text { FEAR }\end{array}$ \\
\hline $\begin{array}{l}\text { (bir işte) saç } \\
\text { sakal ağartmak }\end{array}$ & $\begin{array}{l}\text { MM: o işte uzun zaman çalışmış, } \\
\text { emek vermiş olmak (to work on } \\
\text { and struggle for something) }\end{array}$ & $\begin{array}{l}\text { WHITE HAIR AND } \\
\text { WHITE BEARD } \\
\text { STAND FOR BEING } \\
\text { EXPERIENCED }\end{array}$ \\
\hline $\begin{array}{l}\text { (bir işten) yüz } \\
\text { (yüzünün) akılla } \\
\text { çımak }\end{array}$ & $\begin{array}{l}\text { MM: bir işi kendi saygınlığını } \\
\text { yitirmeden eksiksiz ve başarılı } \\
\text { olarak yapıp bitirmek (acquit } \\
\text { oneself well) }\end{array}$ & $\begin{array}{l}\text { WHITE FACE STANDS } \\
\text { FOR PRIDE }\end{array}$ \\
\hline $\begin{array}{l}\text { (birinin) yüzünü } \\
\text { ăgartmak }\end{array}$ & $\begin{array}{l}\text { MM: beğenilir iş yapmak, iş } \\
\text { ve davranışlarıyla yakınlarının } \\
\text { övünmesine sebep olmak (to make } \\
\text { proud) }\end{array}$ & $\begin{array}{l}\text { WHITE FACE STANDS } \\
\text { FOR PRIDE }\end{array}$ \\
\hline $\begin{array}{l}\text { bembeyaz } \\
\text { kesilmek } \\
\text { (olmak) }\end{array}$ & $\begin{array}{l}\text { MM: beklemediği bir durum } \\
\text { karşısında beti benzi atmak (to } \\
\text { become pale because of being } \\
\text { shocked or scared) }\end{array}$ & FEAR IS WHITE \\
\hline
\end{tabular}

Table 1. Conceptual metaphors/metonymies of white colour idioms in Turkish 


\begin{tabular}{|c|c|c|}
\hline Idiom & Metaphorical Meaning (MM) & $\begin{array}{l}\text { Conceptual Metaphor/ } \\
\text { Metonymy }\end{array}$ \\
\hline $\begin{array}{l}\text { a son of the white } \\
\text { hen }\end{array}$ & MM: a lucky one & $\begin{array}{l}\text { BEING LUCKY IS } \\
\text { WHITE }\end{array}$ \\
\hline a white elephant & $\begin{array}{l}\text { MM: an expensive and useless } \\
\text { luxury }\end{array}$ & $\begin{array}{l}\text { USELESSNESS IS } \\
\text { WHITE }\end{array}$ \\
\hline a white knight & $\begin{array}{l}\text { MM: a party voluntarily coming to } \\
\text { the assistance of another party at a } \\
\text { considerable cost to itself }\end{array}$ & $\begin{array}{l}\text { HELPFULNESS IS } \\
\text { WHITE }\end{array}$ \\
\hline a white lie & $\begin{array}{l}\text { MM: a false statement uttered in a } \\
\text { good cause }\end{array}$ & $\begin{array}{l}\text { HARMLESSNESS IS } \\
\text { WHITE }\end{array}$ \\
\hline a whited sepulchre & $\begin{array}{l}\text { MM: a hypocrite; someone who is } \\
\text { ostensibly virtuous but inwardly } \\
\text { corrupt, literary }\end{array}$ & HYPOCRISY IS WHITE \\
\hline at white heat & MM: of intense passion & PASSION IS WHITE \\
\hline big white chief & $\begin{array}{l}\text { MM: a person in authority, } \\
\text { humorous }\end{array}$ & AUTHORITY IS WHITE \\
\hline to bleed white & $\begin{array}{l}\text { MM: extort the last penny from } \\
\text { someone }\end{array}$ & EXTORTION IS WHITE \\
\hline lily-white & MM: honest and incorruptible & HONESTY IS WHITE \\
\hline lint-white & MM: very white & PALENESS IS WHITE \\
\hline $\begin{array}{l}\text { to look like a } \\
\text { whitewashed wall }\end{array}$ & MM: pale-faced & PALENESS IS WHITE \\
\hline $\begin{array}{l}\text { to mark something } \\
\text { with a white stone }\end{array}$ & $\begin{array}{l}\text { MM: regard something as } \\
\text { especially fortunate or happy }\end{array}$ & HAPPINESS IS WHITE \\
\hline men in white coats & $\begin{array}{l}\text { MM: psychiatrists or psychiatric } \\
\text { workers }\end{array}$ & $\begin{array}{l}\text { A WHITE COAT } \\
\text { STANDS FOR A } \\
\text { PERSON ENGAGED IN } \\
\text { PSYCHIATRIC WORK } \\
\end{array}$ \\
\hline pale/white as whey & MM: pale & PALENESS IS WHITE \\
\hline pearly whites & MM: a person's teeth & LIGHT IS WHITE \\
\hline $\begin{array}{l}\text { to stand in white } \\
\text { sheets }\end{array}$ & MM: make public apology & $\begin{array}{l}\text { WHITE SHEETS STAND } \\
\text { FOR MAKING PUBLIC } \\
\text { APOLOGY }\end{array}$ \\
\hline $\begin{array}{l}\text { to show the white } \\
\text { feather }\end{array}$ & MM: to indicate cowardice & FEAR IS WHITE \\
\hline to turn white & $\begin{array}{l}\text { MM: to express shock at } \\
\text { unexpected news }\end{array}$ & FEAR IS WHITE \\
\hline $\begin{array}{l}\text { to white ant } \\
\text { someone }\end{array}$ & $\begin{array}{l}\text { MM: to surreptitiously seek to } \\
\text { destroy a person }\end{array}$ & $\begin{array}{l}\text { DESTROYING A } \\
\text { PERSON IS WHITE }\end{array}$ \\
\hline to whitewash & $\begin{array}{l}\text { MM: to exonerate when this is not } \\
\text { warranted by the facts }\end{array}$ & $\begin{array}{l}\text { EXAGGERATION IS } \\
\text { WHITE }\end{array}$ \\
\hline
\end{tabular}




\begin{tabular}{|c|c|c|}
\hline Idiom & Metaphorical Meaning (MM) & $\begin{array}{l}\text { Conceptual Metaphor/ } \\
\text { Metonymy }\end{array}$ \\
\hline $\begin{array}{l}\text { white about the } \\
\text { gills }\end{array}$ & $\begin{array}{l}\text { MM: looking depressed or flushed } \\
\text { with anger, drink or indignation, } \\
\text { frightened, sickly }\end{array}$ & BEING SICK IS WHITE \\
\hline white as a clout & MM: pale white & PALENESS IS WHITE \\
\hline white as a doll & MM: bright & LIGHT IS WHITE \\
\hline white as a dove & MM: bright & LIGHT IS WHITE \\
\hline white as a fish & MM: pale-faced & PALENESS IS WHITE \\
\hline $\begin{array}{l}\text { white as a flock of } \\
\text { sheep }\end{array}$ & MM: very white & LIGHT IS WHITE \\
\hline white as a ghost & $\begin{array}{l}\text { MM: extremely pale, as if } \\
\text { frightened }\end{array}$ & PALENESS IS WHITE \\
\hline $\begin{array}{l}\text { white as a hound's } \\
\text { tooth }\end{array}$ & MM: very white & LIGHT IS WHITE \\
\hline white as a kerchief & MM: pale face & PALENESS IS WHITE \\
\hline white as a pillow & MM: extremely pale & PALENESS IS WHITE \\
\hline white as a sheet & $\begin{array}{l}\text { MM: extremely pale, as if } \\
\text { frightened }\end{array}$ & PALENESS IS WHITE \\
\hline white as a spirit & MM: extremely pale & PALENESS IS WHITE \\
\hline white as a statue & MM: extremely pale & PALENESS IS WHITE \\
\hline white as a witch & MM: extremely pale & PALENESS IS WHITE \\
\hline white as ivory & MM: bright & LIGHT IS WHITE \\
\hline white as milk & MM: clean, very white & $\begin{array}{l}\text { CLEANLINESS IS } \\
\text { WHITE }\end{array}$ \\
\hline white as salt & MM: extremely pale & PALENESS IS WHITE \\
\hline white as silver & MM: bright & LIGHT IS WHITE \\
\hline $\begin{array}{l}\text { white as the driven } \\
\text { snow }\end{array}$ & $\begin{array}{l}\text { MM: extremely pale, as if } \\
\text { frightened }\end{array}$ & PALENESS IS WHITE \\
\hline $\begin{array}{l}\text { white as } \\
\text { whalebone }\end{array}$ & MM: very white & BEAUTY IS WHITE \\
\hline white crow & MM: rarity & RARITY IS WHITE \\
\hline white flag & MM: token of surrender & $\begin{array}{l}\text { WHITE FLAG STANDS } \\
\text { FOR PEACE }\end{array}$ \\
\hline white hen's chick & MM: spoilt, petted child & \begin{tabular}{|l} 
BEING SPOILT IS \\
WHITE
\end{tabular} \\
\hline white knuckle & $\begin{array}{l}\text { MM: something to survive } \\
\text { something threatening through } \\
\text { strained endurance }\end{array}$ & ENDURANCE IS WHITE \\
\hline white land & $\begin{array}{l}\text { MM: land where no further } \\
\text { developments will be allowed }\end{array}$ & $\begin{array}{l}\text { LAND WHERE NO } \\
\text { DEVELOPMENT IS } \\
\text { ALLOWED IS WHITE }\end{array}$ \\
\hline
\end{tabular}




\begin{tabular}{|c|c|c|}
\hline Idiom & Metaphorical Meaning (MM) & $\begin{array}{l}\text { Conceptual Metaphor/ } \\
\text { Metonymy }\end{array}$ \\
\hline white livered & MM: cowardly, spiritless & $\begin{array}{l}\text { WHITE LIVER STANDS } \\
\text { FOR FEAR }\end{array}$ \\
\hline white trash & $\begin{array}{l}\text { MM: an offensive way of } \\
\text { describing poor white people who } \\
\text { are not educated }\end{array}$ & $\begin{array}{l}\text { WHITE TRASH STANDS } \\
\text { FOR AN UNEDUCATED } \\
\text { AND POOR PERSON }\end{array}$ \\
\hline white-bread & $\begin{array}{l}\text { MM: white-bread people or things } \\
\text { are ordinary and boring, and often } \\
\text { those that are typical of white, } \\
\text { American people }\end{array}$ & $\begin{array}{l}\text { WHITE-BREAD STANDS } \\
\text { FOR AN ORDINARY } \\
\text { AND BORING PERSON }\end{array}$ \\
\hline white-collar & $\begin{array}{l}\text { MM: a white-collar worker is } \\
\text { someone who works in an office, } \\
\text { doing mental rather than physical } \\
\text { work }\end{array}$ & $\begin{array}{l}\text { WHITE COLLAR } \\
\text { STANDS FOR PERSON } \\
\text { PERFORMING NON- } \\
\text { MANUAL WORK }\end{array}$ \\
\hline whiter than white & $\begin{array}{l}\text { MM: extremely white, morally } \\
\text { beyond reproach }\end{array}$ & HONESTY IS WHITE \\
\hline with white hands & $\begin{array}{l}\text { MM: innocently, honestly, without } \\
\text { guilt }\end{array}$ & $\begin{array}{l}\text { WHITE HAND STANDS } \\
\text { FOR HONESTY }\end{array}$ \\
\hline
\end{tabular}

Table 2. Conceptual metaphors/ metonymies of white colour idioms in English

As for the commonalities, the cognitive analysis of Turkish and English white colour idioms demonstrated that the white colour most frequently refers to honesty which is observed in ak süt, ak süt emmiş, yüzü ak, sütten çıkmış ak kaşık gibi olmak, ak yüzlü, alnı açık yüzü ak, and anasının ak sütü in Turkish. In 4 of these idiomatic expressions (ananın ak sütü gibi, ak süt, sütten çıkmış ak kaşık gibi olmak, and ak süt emmiş), white is collocated with 'milk' of a mother. The holiness of mother's milk is always emphasized in Turkish mythology, and the Sakha Yakut Turks believed that the goddess of motherhood, Ayzit, gave life to her baby with her breast milk (Samur 2008: 7). What is more, in alnı açık yüzü $a k$, yüzü $a k$, and ak yüzlü, white is collocated with 'face' generating the conceptual metonymy WHITE FACE STANDS FOR HONESTY.

Honesty is also embedded in the minds of English speakers with the colour white as observed in the idioms lily-white, with white hands, and whiter than white. In these idioms, white connotes being honest and incorrupt as inferred from their meanings and naturally occurring examples.

Furthermore, the colour white most frequently refers to light in English as in white as ivory, white as silver, white as a doll, white as a dove, white as a flock of sheep and white as a hound's tooth. Similarly, in Turkish, white is associated 
with the day light as observed in gün ăgarmak, ortallk ağarmak, tan ăgarmak, and tan yeri ăgartmak.

Cleanliness is conceptualized in the two cultures through the colour white as observed in ak pak, beyaza çekmek, kar beyaz, and süt beyaz in Turkish and white as milk in English. Other than creating OLDNESS IS WHITE conceptual metaphor, the idiom akpak generates the CLEANLINESS IS WHITE conceptual metaphor which can be determined depending on the context.

Furthermore, white is also associated with the emotion of fear in Turkish and English, as fear causes a change in the colour of the skin as exemplified in bembeyaz kesilmek and yüzü kireç gibi olmak in Turkish. However, the association of white and cowardice (a type of fear) is more cultural in English as revealed in to show white feather and white-livered. For instance, for white feather, it was pointed out that as pure-bred cock has no white feather, a cock with a white feather in its tail is underbred and is believed to perform poorly in terms of breeding and fighting cocks (Flavell, Flavell 1992: 84). Thus, showing a white feather is considered to be a sign of cowardice in English.

As for the differences, paleness is one of the most striking varieties between the conceptualization of English and Turkish speakers. While paleness is the most frequently observed domain in English, it is not conceptualized in Turkish. White is used to describe the physical appearance of things and refers to the paleness of the skin in English as in white as a sheet, white as a ghost, white as the driven snow, white as a kerchief, look like a whitewashed wall, white as whey, white as a fish, white as salt, white as a pillow, white as a clout, white as a witch, white as a spirit, lint-white, and white as a statue.

What is more, while oldness is the most frequently observed target domain in Turkish, it is never conceptualized through the colour white in English, despite the fact that the whitening of hair, beard, and moustache is a physiological fact. The conceptual metonymy WHITE BEARD STANDS FOR OLDNESS is observed in a $k$ sakaldan yok sakala gelmek, sakalına ak düşmek, ak sakallı, and there is the conceptual metonymy of WHITE HAIR STANDS FOR OLDNESS in saçına ak düşmek. Again associated with oldness, ak sakaldan yok sakala gelmek refers to powerlessness because of aging and ak sakall also refers to wisdom with old age. What is more, the experience and knowledge of old people are foregrounded in Turkish idioms saç ăgartmak, saçı değirmende ă̆artmamak, sakalı değirmende ağartmamak, (bir işte) saç sakal ăgartmak.

Pride is another recurring target domain which is conceptualized by Turkish speakers as observed in the expressions of alninın akıyla generating WHITE FOREHEAD STANDS FOR PRIDE conceptual metonymy. Also in (bir işten) yüz (yüzünün) akıyla çıkmak, yüz akı and (birinin) yüzünü ăgartmak, the white face symbolizes pride in Turkish culture. 


\section{Conclusion}

In this study, white colour idioms in Turkish and English were analysed within the framework of CMT and it was revealed that the differences $(n=29)$ between the connotations of white in these idioms outnumber the commonalities $(n=12)$ among these languages. Despite the common associations of the colour white which are light, honesty, innocence, fear, beauty, good luck, evil, happiness, harmlessness, non-manual work, cleanliness, and peace, it was observed that the colour white refers to cash, oldness, anger, wisdom, pride, heroin, electricity, agreement, hope, purity, white clothes, being experienced, and new page in Turkish, while it connotes uselessness, helpfulness, hypocrisy, passion, authority, extortion, psychiatric work, public apology, rarity, being spoilt, endurance, uneducated and poor person, ordinary and boring, paleness, exaggeration, and sickness in English. These results demonstrated that collocational realizations of the colour white are mostly culturally-oriented and these dissimilarities originate from the different cultural, historical, and social backgrounds of these languages. In other words, this study proved the fact that although Turkish and English speakers see the colour white in the same way, what they perceive and how they conceptualize the world through this colour mostly bear differences.

\section{References:}

Aksoy 1998: Aksoy Ö. A. Deyimler Sözlüğü II. İstanbul: İnkılap Yayınları.

Berlin, Kay 1969: Berlin B., P. Kay. Basic Color Terms: Their Universality and Evolution. Berkeley: University of California Press.

Butterworth et al. 2002: Butterworth A., Gurney D., Malfroy E., G. Mark. Cambridge International Dictionary of Idioms. UK: Cambridge University Press.

Chielens 2007: Chielens L. Basic Colour Terms in English: An Examination of their Use and Meaning in English Expressions. Diss. Universiteit Gent.

Eminoğlu 2014: Eminoğlu H. Türkçe'de Renkler Sözlüğü. Ankara: Gazi Kitabevi

Flavell, Flavell 1994: Flavell L., R. Flavell. Dictionary of Idioms and Their Origins. London: Kayle Cathie.

Gibbs, Steen 1999: Gibbs R., G. Steen (Eds.) Metaphors in Cognitive Linguistics. Amsterdam: John Benjamins.

Hastürkoğlu 2017: Hastürkoğlu G. A Corpus-Based Cognitive Study on the Comparison of Collocational Realizations of Basic Colour Terms in Turkish and English. PhD thesis (unpublished)

He 2011: He G. A Comparative Study of Color Metaphors in English and Chinese. - Theory and Practice in Language Studies. 1.12. 1804-1808. $<$ https://pdfs.semanticscholar.org/08 fb/9995682801a456dd80d4eb9f6335d82e4d58.pdf > [Accessed 15 Sept. 2017]

Lakoff, Johnson 1980: Lakoff G., M. Johnson. Metaphors We Live By. Chicago, London: The University of Chicago Press. 
Philip 2006: Philip G. S. Connotative Meaning in English and Italian Colour-Word Metaphors. - Metaphorik. vol. 10, 59-93. <http://www.metaphorik.de/sites/www. metaphorik.de/files/journal-pdf/10_2006_philip.pdf > [Accessed 15 Oct. 2017]

Rasekh, Ghafel 2011: Rasekh A. E., B. Ghafel. Basic Colors and Their Metaphorical Expressions in English and Persian: Lakoff's Conceptual Metaphor Theory in Focus. - 1st International Conference on Foreign Language Teaching and Applied Linguistics. $<$ http://eprints.ibu.edu.ba/33/1/FLTAL\%202011\%20Proceed\%C4\%B1ngs\%20Book_1_ p211-p224.pdf $>$. [Accessed 15 Sept. 2017]

Potter et al. 1997: Potter E., Watson J., Lax M., Timewell M., J. Todd (eds.). Collins Cobuild Dictionary of Idioms. Harper Collins Publishers.

Renton 1990: Renton N. E. Metaphorically Speaking: A Dictionary of 3800 Picturesque Idiomatic Expressions. Melbourne, New York: Warner Books.

Samur 2008: Samur G. Anne sütü. Ankara, Ministry of Health. $<$ http://www.annevebebek. gov.tr/uploads/dokumanlar/anne_sutu_20.pdf $>$. [Accessed 12 Oct. 2017].

Siefring 2004: Siefring J. (ed.). Oxford Dictionary of Idioms. New York: Oxford University Press.

Spears 2005: Spears R. McGraw-Hill's Dictionary of American Idioms and Phrasal Verbs. USA: McGraw-Hill.

Topçu 2001: Topçu N. Fransizca ve Türkçe Renk İsimlerini İçeren Deyimlerin Karşılaştırmalı İncelemesi. - Hacettepe University Journal of Educational Sciences. 20. 131-140. <http://dergipark.ulakbim.gov.tr/hunefd/article/view/5000048891>. [Accessed 15 Oct. 2017].

Türk Dil Kurumu. Atasözleri ve Deyimler Sözlügüu. <http://tdk.gov.tr/>. [Accessed 15 Oct. 2017].

Wilkinson 2002: Wilkinson P.R. Thesaurus of Traditional English Metaphors. London: Routledge.

Yörük, Yörük 1997: Yörük S., Y. Yörük. Açıklamalı Deyimler Sözlüğü. İstanbul: Serhat Yayınları. 